\title{
Application of Non-stationary EIV Methods to Transient Electromagnetic Mineral Exploration
}

\author{
K. Lau, J. H. Braslavsky, J. C. Agüero and G. C. Goodwin* \\ * ARC Centre for Dynamic Systems and Control, The University of \\ Newcastle, Callaghan, NSW, 2308,AUSTRALIA. \\ $\{$ K.Lau, Julio.Braslavsky, Juan.Aguero, \\ Graham.Goodwin\}@newcastle.edu.au
}

\begin{abstract}
In this paper, we apply a non-stationary errors-in-variables model estimation technique to a problem arising in transient electromagnetic mineral exploration. The proposed technique is used to estimate a model which is deployed for noise cancellation. Alternative methods for noise cancellation in these systems rely on specific signal characteristics, and are thus not readily transferable to other applications. The proposed technique produces an estimated model that agrees well with those obtained using alternative methods, and achieves noise reduction levels similar to those achieved via the alternative methods. This is shown by performance comparisons on experimental data. An advantage of the proposed technique is that it is more readily transferable to other applications.
\end{abstract}

\section{INTRODUCTION}

An errors-in-variables (EIV) system is one in which both the input and output are subject to measurement noise. A typical EIV system is shown in Fig. 1. From Fig. 1, we have

$$
\begin{aligned}
& u(k)=u_{0}(k)+n_{1}(k), \\
& y(k)=y_{0}(k)+n_{2}(k),
\end{aligned}
$$

where $u(k)$ and $y(k)$ are the measured input and output, $u_{0}(k)$ and $y_{0}(k)$ are the true input and output, and $n_{1}(k)$ and $n_{2}(k)$ are the measurement noise sequences. The transfer function from $u_{0}(k)$ to $y_{0}(k)$ is denoted by $G(z)$.

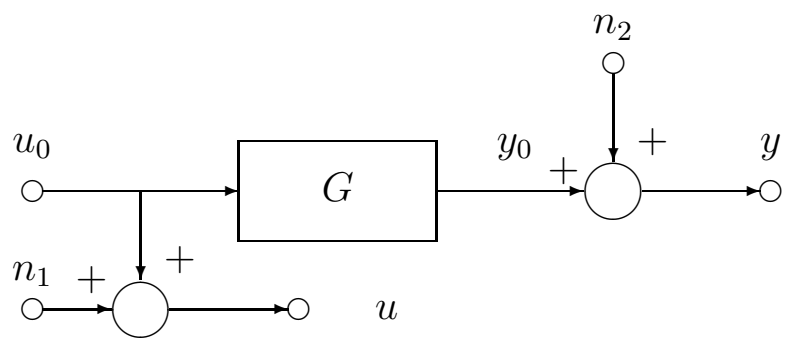

Fig. 1. An errors-in-variables system.

It is well known that when the noise parameters are unknown, EIV models are not identifiable from second order properties [Agüero and Goodwin, 2008b]. The identifiability problem can be resolved by imposing additional assumptions (e.g., knowledge of the ratio of the noise variances). A recent review of EIV identification techniques, and the assumptions required in each case is given in Söderström [2006].

In this paper, we focus on the application of EIV methods to a model estimation problem encountered in transient electromagnetic (TEM) mineral exploration (Carter [2005], Kearney et al. [2002], Nabighian and Macnae [1991] and Geophysical Exploration for Engineers [1998]). Errorsin-variables issues arise in this problem due to the nature of the input signal. The authors in a recent paper [Lau et al., 2007] proposed a method for mitigating the EIV problem by utilising specific knowledge of the input signal characteristics. However, these characteristics are problem specific and thus the associated approach is not readily transferable to other applications.

In the current paper, we explore the application of a different procedure which utilises less prior knowledge and which is thus more readily transferable to other applications. In particular, we will exploit non-stationarity features of the data.

The use of non-stationary data to resolve EIV problems has a long history for the static case [Wald, 1940]. More recently, Markovsky et al. [2006] have extended the idea to special cases of the dynamic problem. These results are also discussed in the recent review paper by Söderström [2006]. Further extensions are discussed in the companion paper to the current paper [Agüero and Goodwin, 2008a].

In the present paper, we will study the application of one of the techniques discussed in Agüero and Goodwin [2008a] to the TEM problem. The results are validated against models found using two independent methods (including the one proposed in Lau et al. [2007]). It is shown that the estimated response found by the method used here agrees well with those found using the alternative methods. In addition, the fitted model performs equally well as the 
validation models when used for noise cancellation. This indicates that the technique can be successfully applied in this case, and suggests that the approach could be useful in other EIV applications.

The layout of the remainder of the paper is as follows: In Sect. 2, we provide background information on TEM surveying and sferics noise. Then, in Sects. 3 and 4 , we introduce the EIV problem and describe the nonstationary EIV method that we will subsequently deploy. We apply the EIV method and validate the estimated models in Sect. 5. Conclusions are drawn in Sect. 6.

\section{BACKGROUND TO TEM SURVEYING AND SFERICS}

\subsection{TEM surveying}

Transient electromagnetic surveying (also known as timedomain electromagnetic surveying) is a technique used in mineral exploration to detect underground conductive ore bodies by induction and detection of electromagnetic (EM) fields. Here we will be concerned with ground-based transient electromagnetic (TEM) surveying using an Australian-developed technology known as Geoferret $^{\mathrm{TM}}$ [Carter, 2005]. Fig. 2 shows a typical system configuration for TEM surveying using this system.

The typical operation of a TEM surveying system consists of two phases: firstly, the transmission of a primary field, during which no measurements are made, and secondly, after the transmitter is switched off, the detection of the secondary field response (i.e., the transient response) of the earth. To generate the primary field, a pulsed current waveform is passed through a loop or coil of wire (the transmitter), which is laid on the surface of the area to be surveyed. The primary field induces superficial underground eddy currents, which in turn induce eddy currents at greater depths in any conductive ore bodies. These underground decaying currents produce the secondary field, which can be measured by the array of receiving antennae on the earth's surface. The magnitude and rate of decay of the secondary field depends on the electrical conductivity of the ground, and, through posterior signal processing, allows the identification and location of target ore bodies at depths of up to $500 \mathrm{~m}$.

We consider a system in which a periodic current waveform consisting of a series of square pulses of alternating sign is used. The secondary field at different locations is measured using a number of receiver coils. The measured data is then interpreted to obtain a geological model which is consistent with the data.

A more detailed description of TEM surveying can be found in Kearney et al. [2002], Nabighian and Macnae [1991], and Geophysical Exploration for Engineers [1998].

The measured responses in TEM surveying often have low signal-to-noise ratios. The sources of noise include instrument noise and environmental noise (including 50 $\mathrm{Hz}$ power line interference). In the next section we describe an important source of environmental noise referred to as 'sferics'.
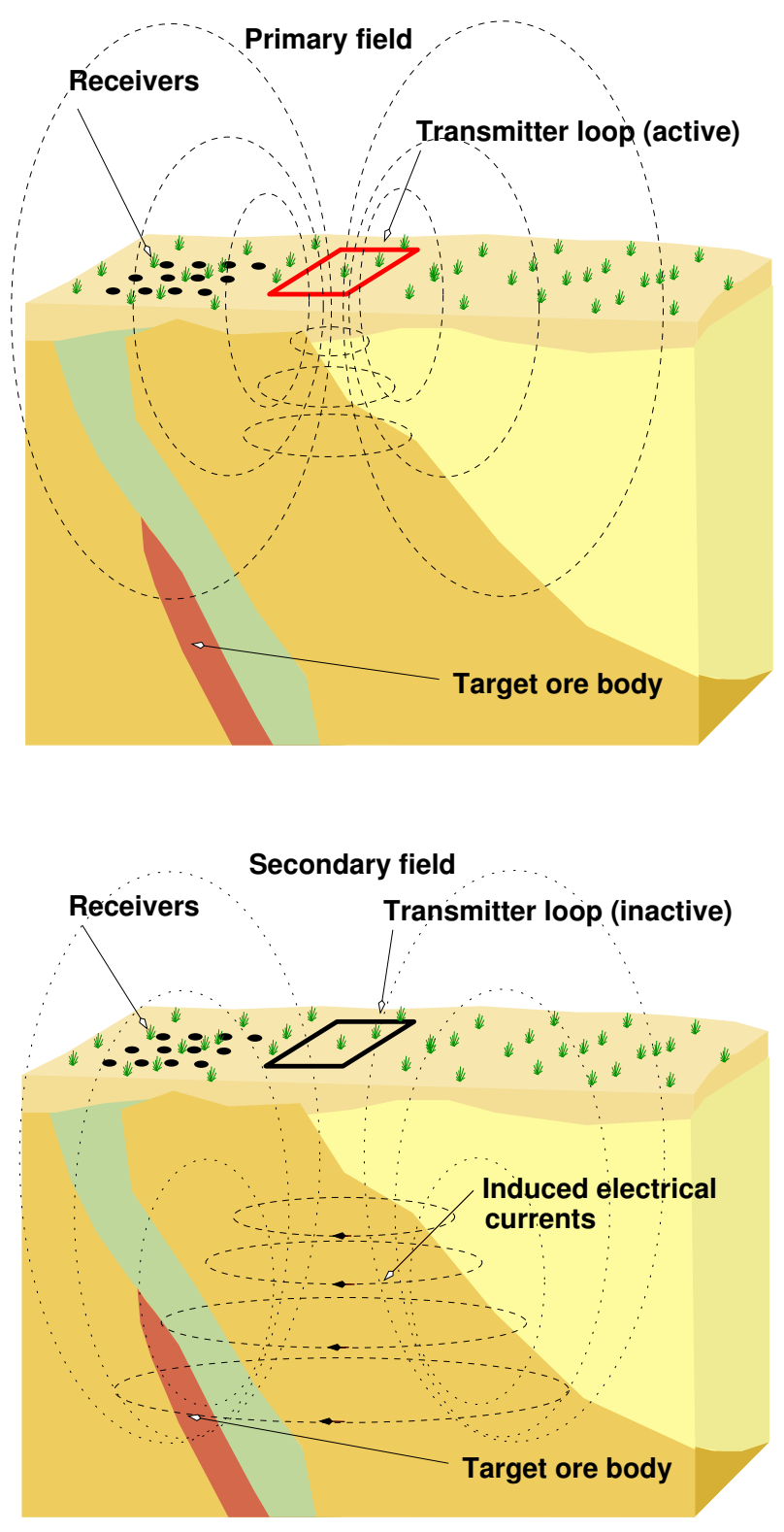

Fig. 2. Typical ground-based TEM surveying system configuration.

\subsection{Sferics}

A major contributor to noise in the detection of deep underground ore bodies is sferics: environmental EM radiation that dominates receiver instrument noise in some environments (such as close to the equator). Sferics (short for 'atmospherics') originate from the EM radiation produced by lightning strikes. These EM signals can travel thousands of kilometres through the space between the earth and the ionosphere, which acts as a waveguide. Hence, local and distant lightning storms contribute to the sferics noise measured at any single point.

Sferics noise can be divided into two groups, local and distant. Local sferics noise consists of large, infrequent, bursts of short pulses (i.e., it is impulsive), and is nonstationary in nature. Fig. 3 shows a measured large sferic occurring at approximately 0.002 s. Distant sferics noise 
may be characterised as essentially coloured noise. It has been estimated that there are approximately 100 lightning strikes per second worldwide. Thus, distant sferics noise consists of many small pulses and can be considered to be quasi-stationary. The spectrum of sferics noise is concentrated in the $1-500 \mathrm{~Hz}$ and $2.5-10 \mathrm{kHz}$ frequency bands. The dip in the spectrum between $500 \mathrm{~Hz}$ and 2.5 $\mathrm{kHz}$ is due to the attenuation of the earth-ionosphere waveguide at these frequencies.

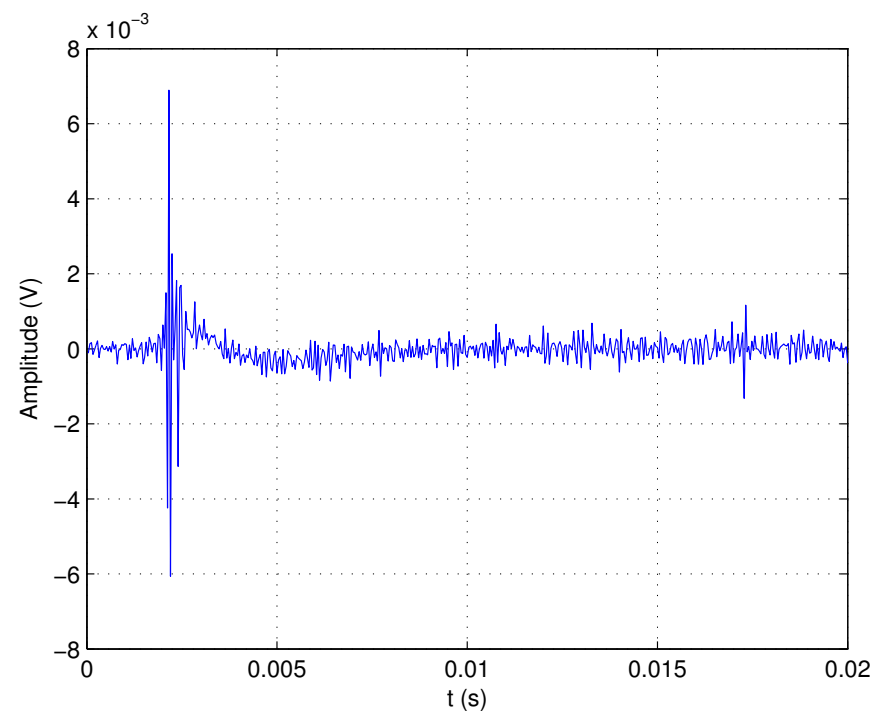

Fig. 3. A measured sferic (output antenna).

\section{SFERICS NOISE CANCELLATION AND EIV}

One way of reducing the effect of sferics is to perform noise cancellation from one antenna to another. The reference antenna is placed at a remote location, so that the effect of the transmitted signal is negligible. The technique utilises the fact that the sferics measured at different locations are affected by local geographical conditions but are correlated.

In order to perform noise cancellation one needs to find a model relating the sferics measured at the reference and output antennae. We do this by estimating a model from measurements taken at the two antennae with the transmitter turned off. The (true) input and output to the model are the sferics components of the measurements taken at the two antennae. Hence, in this context, the sferics are considered as the signals and the rest of the components (instrument noise, $50 \mathrm{~Hz}$ harmonics, etc.) are considered as measurement noise. It follows that the system can be formulated as an EIV system of the form shown in Fig. 1. In our case, $u$ is the measured signal at the reference antenna, $u_{0}$ is the sferics component of this signal, and $n_{1}$ consists of the rest of the signal components. The signals $y, y_{0}$ and $n_{2}$ are defined in a similar manner for the output antenna.

\section{THE NON-STATIONARY EIV METHOD}

As stated in the introduction, we plan to utilise one of the non-stationary EIV techniques outlined in Agüero and Goodwin [2008a]. In particular, we note that the sferics signal is non-stationary due to storm activity. We then utilise the following assumption:

Assumption 1. The spectrum of $u_{0}$ is different in two non-overlapping time periods $\left(t_{1}^{A}, t_{2}^{A}\right)$ and $\left(t_{1}^{B}, t_{2}^{B}\right)$.

Assumption 1, together with the usual assumption that $n_{1}$ and $n_{2}$ are stationary allows the identifiability problem to be resolved as outlined below.

Let $\Phi_{x y}(\omega)$ denote the cross power spectral density (CPSD) of the signals $x$ and $y$, and let $\hat{\Phi}_{x y}(\omega)$ be an estimate of the CPSD. If Assumption 1 holds, then an estimate, $\hat{G}\left(e^{j \omega}\right)$, of the frequency response, $G\left(e^{j \omega}\right)$, is given by [Agüero and Goodwin, 2008a]

$$
\hat{G}\left(e^{j \omega}\right)=\frac{\hat{\Phi}_{y_{A} u_{A}}(\omega)-\hat{\Phi}_{y_{B} u_{B}}(\omega)}{\hat{\Phi}_{u_{A} u_{A}}(\omega)-\hat{\Phi}_{u_{B} u_{B}}(\omega)},
$$

where the subscripts ' $A$ ' and ' $B$ ' denote the parts of the signals corresponding to $t \in\left(t_{1}^{A}, t_{2}^{A}\right)$ and $t \in\left(t_{1}^{B}, t_{2}^{B}\right)$, respectively. Equation (3) can be derived by noting that

$$
\begin{aligned}
& \Phi_{u_{A} u_{A}}(\omega)=\Phi_{u_{0 A} u_{0 A}}(\omega)+\Phi_{n_{1} n_{1}}(\omega), \\
& \Phi_{y_{A} u_{A}}(\omega)=G\left(e^{j \omega}\right) \Phi_{u_{0 A} u_{0 A}}(\omega), \\
& \Phi_{u_{B} u_{B}}(\omega)=\Phi_{u_{0 B} u_{0 B}}(\omega)+\Phi_{n_{1} n_{1}}(\omega), \\
& \Phi_{y_{B} u_{B}}(\omega)=G\left(e^{j \omega}\right) \Phi_{u_{0 B} u_{0 B}}(\omega) .
\end{aligned}
$$

\section{APPLICATION OF THE NON-STATIONARY EIV METHOD TO MODEL ESTIMATION FOR SFERICS NOISE CANCELLATION}

In this section, we use the non-stationary EIV method described in the preceding section to estimate the model from the reference antenna to the output antenna.

The method is tested using two $60 \mathrm{~s}$ intervals of data that have noticeable differences in spectra. The data is sampled at a rate of $25 \mathrm{kHz}$. Let $u_{A}$ and $y_{A}$ correspond to the first interval of the data and let $u_{B}$ and $y_{B}$ correspond to the second interval.

Fig. 4 shows the PSDs of $u_{A}, u_{B}, y_{A}$ and $y_{B}$. We observe that the PSDs have a large noise mound between approximately 10 and $600 \mathrm{~Hz}$. We focus on reducing this mound because it is known that sferics have significant power in this frequency range. It can be seen that there is a difference in the PSDs of sections A and B of the data in the region of interest (i.e., the region containing the mound). This is due to the non-stationary nature of local sferics.

We concentrate on the reduction of the 'baseline' noise spectrum, not the harmonic components as these latter components can be removed separately. For our purpose, the rest of spectrum, i.e., the harmonic disturbances and the parts of the spectrum above approximately $600 \mathrm{~Hz}$ and below approximately $10 \mathrm{~Hz}$ are treated as measurement noise. More detailed descriptions of these parts of the spectrum can be found in Lau et al. [2007].

Fig. 5 shows the magnitude of the estimated frequency response when the non-stationary EIV method (Equation $(3))$ is used to calculate the response. The response is shown between 10 and $600 \mathrm{~Hz}$ for the reasons discussed 
above. The figure also shows the (biased) estimate obtained by ignoring the measurement noise on the input and using

$$
\hat{G}\left(e^{j \omega}\right)=\frac{\hat{\Phi}_{y u}(\omega)}{\hat{\Phi}_{u u}(\omega)}
$$

to calculate the response. We refer to this method of estimating the response as the naive method. From the figure, it can be seen that the EIV and naive methods yield quite different frequency responses. In particular, the naive estimate is less than the EIV estimate (at most frequencies). In the sections that follow, we confirm that the EIV method results in a better model of the system.

We notice that the estimate using the non-stationary EIV method has a much larger variance than the biased (naive) estimate. This is due to the ratio calculated in (3) and due to harmonic components. The EIV method assumes that the CPSDs and PSDs of the harmonic components in sections $\mathrm{A}$ and $\mathrm{B}$ of the data cancel exactly. If the harmonics are large, then small errors in the cancellation can result in large errors in the estimated gain. Since the harmonic disturbances appear as outliers in the (C)PSDs, we remove them by applying a median filter (a form of outlier rejection) to the (C)PSDs. We then fit a first order biproper model to the resulting frequency response.
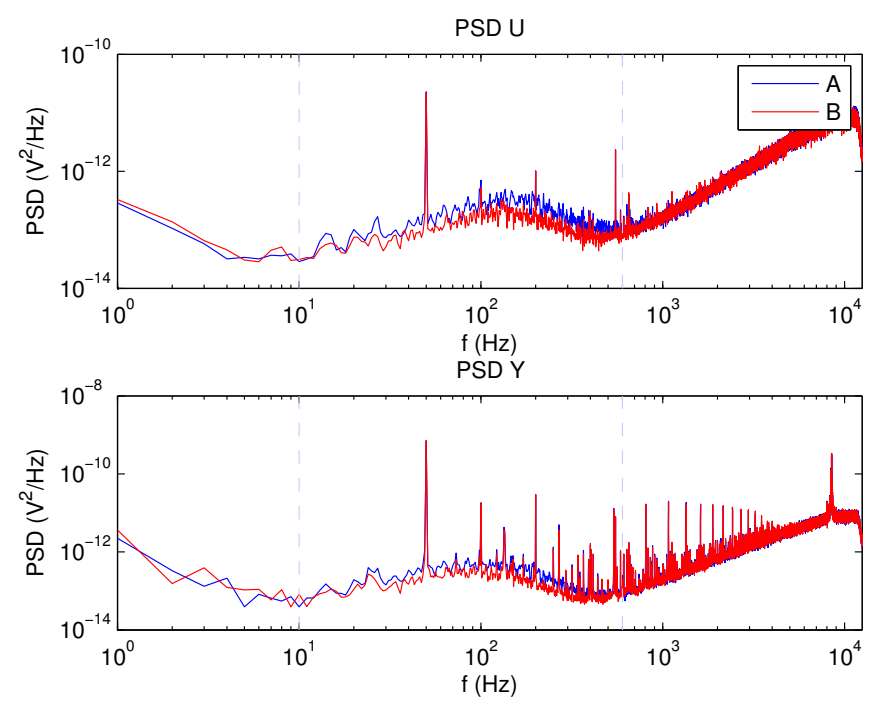

Fig. 4. PSDs of the signals used to estimate the frequency response using the non-stationary EIV method. (Top) PSDs of $u_{A}$ and $u_{B}$. (Bottom) PSDs of $y_{A}$ and $y_{B}$.

\subsection{Comparison to a model found using time and frequency} selectivity

Fig. 6 shows the estimated frequency responses (with the outliers removed) for the EIV and naive methods. The responses of the fitted first order models are also plotted. We validate these responses against a model found using the approach described in Lau et al. [2007] and summarised below. It is clear that the non-stationary EIV method provides a model which is closer to the validation model than the model obtained using the naive method.

The validation model is found by exploiting time and frequency selectivity to isolate a part of the input with a good

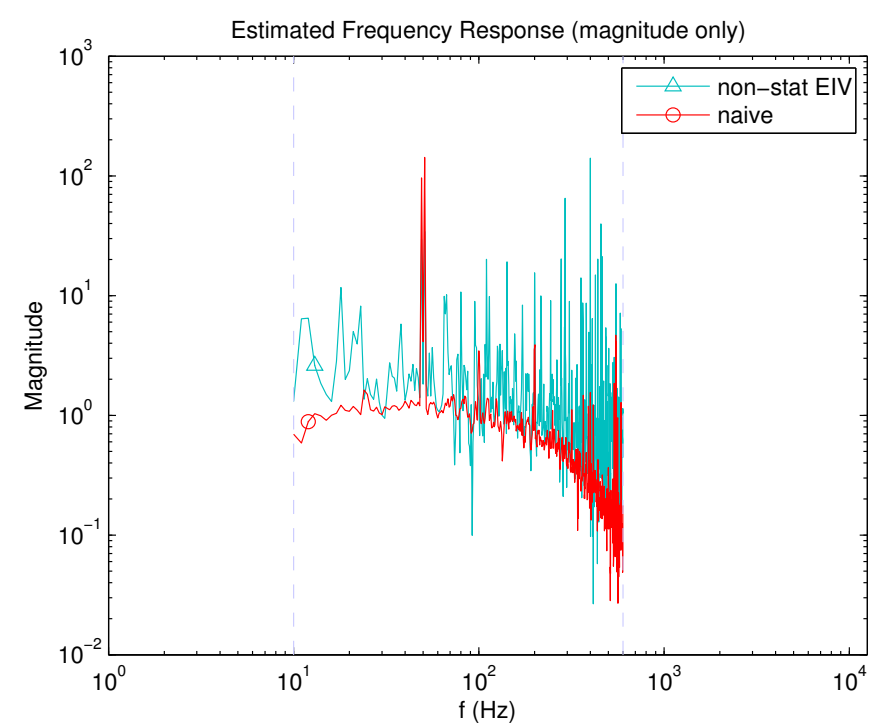

Fig. 5. Estimated frequency responses (magnitude only) using the non-stationary EIV and naive methods.

signal to noise ratio. The procedure can be summarised as follows: A bandpass filter with a passband between 20 and $400 \mathrm{~Hz}$ is applied to the input and output data. In the filtered signals, the sferics appear as large pulses which stand out from the filtered noise. We fit a model to a 'marked sferic', a short section of the filtered data containing a large sferic (and hence, a good signal to noise ratio). We refer to this method of finding a model as the marked data method. More details can be found in Lau et al. [2007]. As noted in the Introduction, this validation method uses specific knowledge regarding this particular application.

\subsection{Comparison to a model found using an independent output measurement}

For a second validation test we utilise measurements from an independent output antenna. Let $v(k)$ be the measured output, $v_{0}(k)$ be the true output, $n_{3}(k)$ be the measurement noise, and $G_{v}(z)$ be the transfer function from $u_{0}(k)$ to $v_{0}(k)$. We have

$$
v(k)=v_{0}(k)+n_{3}(k) .
$$

It can be easily shown that an unbiased estimate of $G\left(e^{j \omega}\right)$ is given by

$$
\hat{G}\left(e^{j \omega}\right)=\frac{\hat{\Phi}_{y v}(\omega)}{\hat{\Phi}_{u v}(\omega)} .
$$

We refer to this method as the independent output method.

The frequency responses obtained using the non-stationary EIV method and independent output method are compared in Fig. 7. We again see that responses found using the EIV method agree well with the validation method.

\subsection{Comparison of noise cancellation performance}

For a final validation test, we compare the noise cancellation performance of the models found using all four of the methods described in this paper (non-stationary EIV, naive, marked data and independent output). The test is 

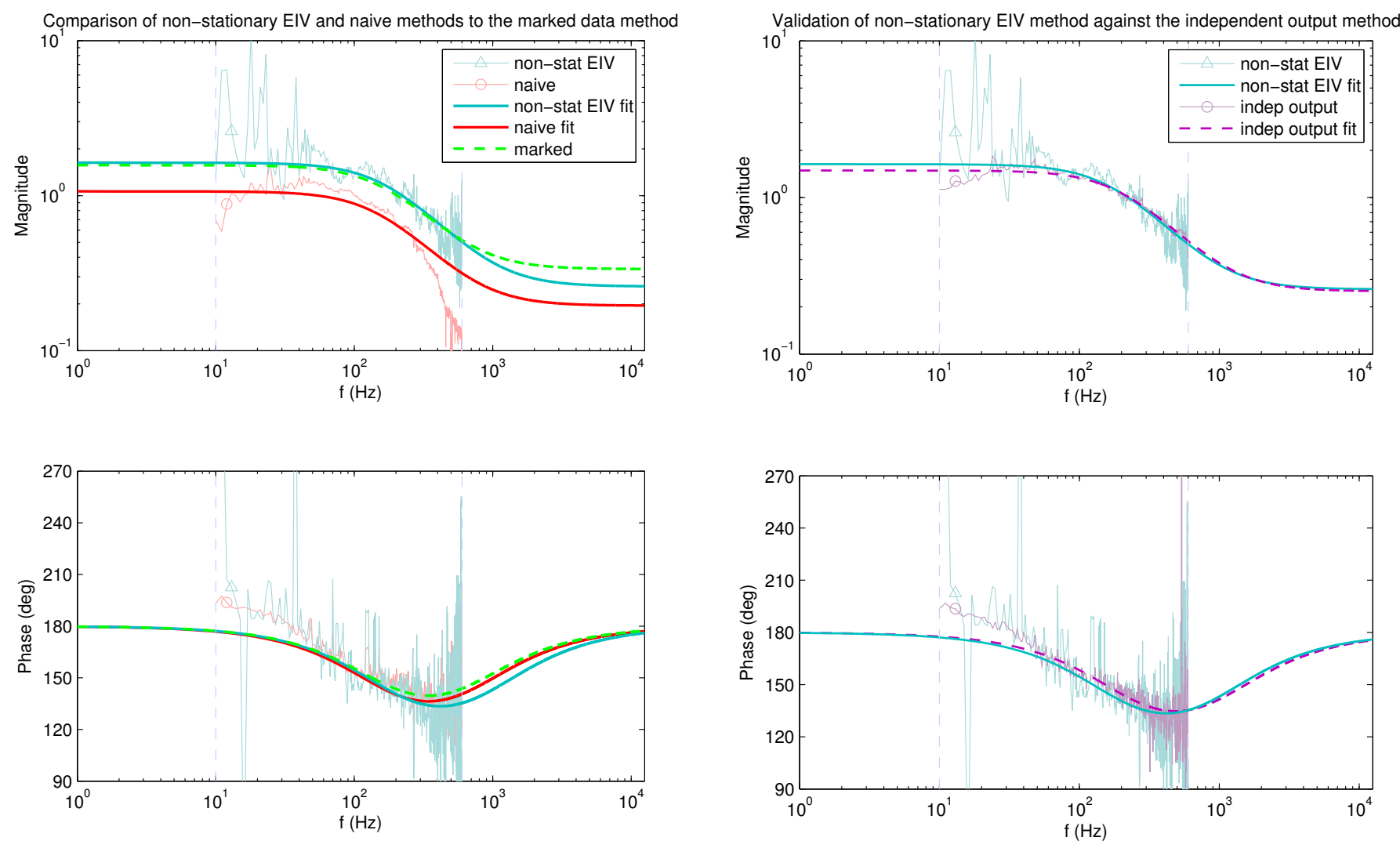

Fig. 6. Comparison of the responses found using the non-stationary EIV and naive methods to the model found using marked data. The estimated frequency responses with outlier rejection and the responses of the fitted models for the non-stationary EIV and naive methods are shown.

performed on a collection of marked sferics taken from a different set of data to that used for fitting the models. The procedure used to obtain each of the marked sferics is the same as that outlined in Sect. 5.1 except that a slightly wider bandpass filter $(10$ to $800 \mathrm{~Hz})$ is used. The sferics are selected using a simple thresholding method.

Noise cancellation is performed by feeding marked sferics obtained at the reference antenna through the models to generate estimates of the marked sferics at the output antenna. The results are illustrated in Fig. 8. The figure shows the marked sferics at the output and the residual errors when the fitted models are used for noise cancellation. The errors are offset from zero to separate the plots. The figure shows that the residual errors for the non-stationary EIV, marked and independent output methods are similar in size, and are noticeably smaller than the residual error for the naive method. Fig. 9 shows the PSDs of the signals shown in Fig. 8, and confirms that the model found using the EIV method performs in a comparable fashion to the two validation models. All three of these models provide a significant reduction in the sferics noise mound.

\section{CONCLUSION}

This paper has applied a non-stationary EIV method to transient electromagnetic data used in mineral exploration. The method has been validated by comparing its

Fig. 7. Validation of the non-stationary EIV method against the independent output method. The estimated frequency responses with outlier rejection and the responses of the fitted models for the two methods are shown.

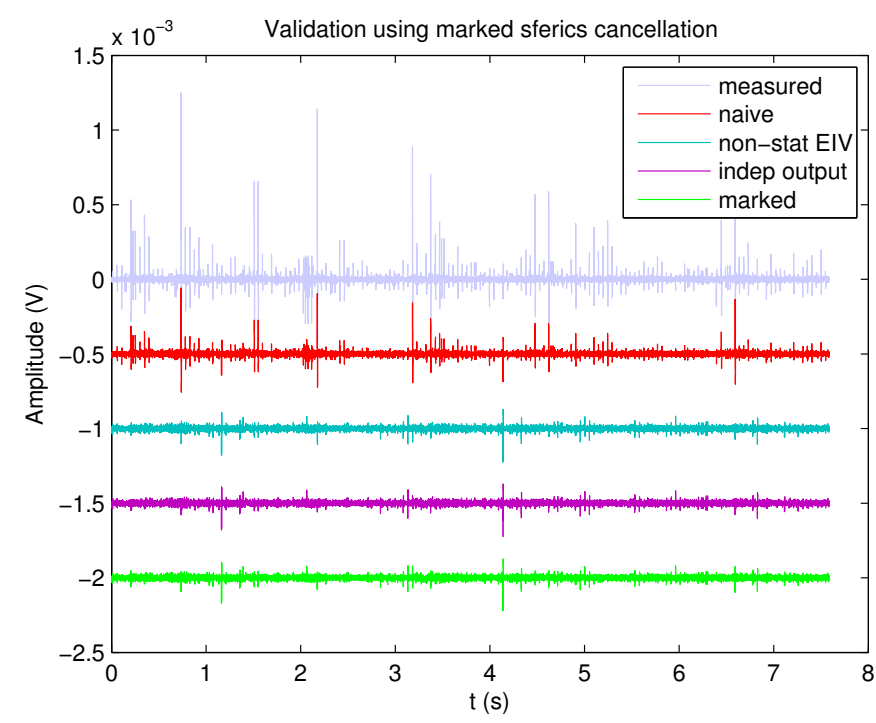

Fig. 8. Filtered sferics at the output (marked sferics) and residual errors when the fitted models are used for noise cancellation.

performance with two other methods (marked data, and independent output) which exploit specific features of the transient electromagnetic problem and are thus difficult to translate to other problems. 


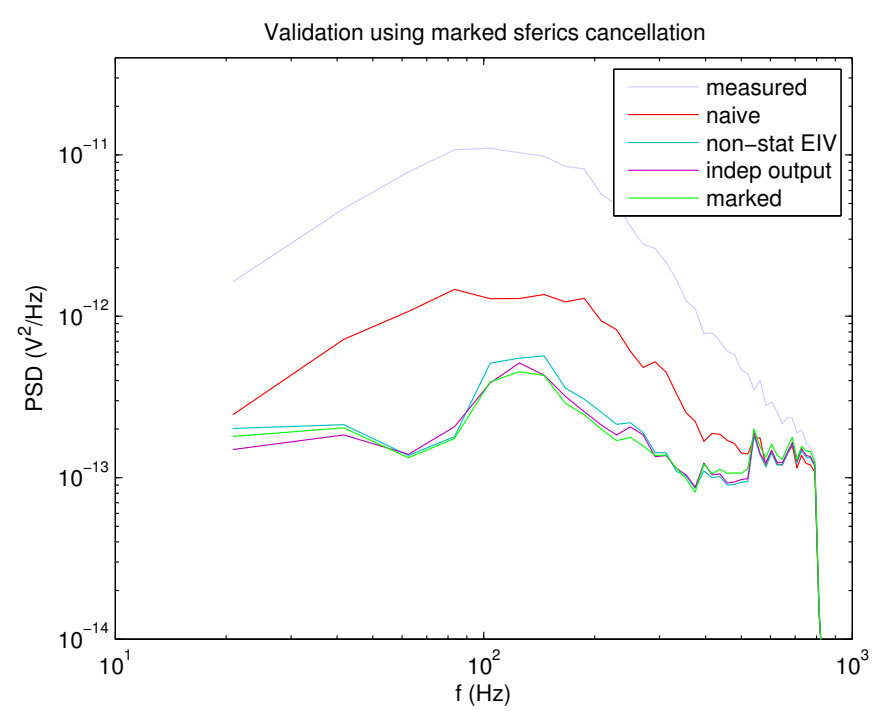

Fig. 9. PSDs of the signals shown in Fig. 8.

All three methods (i.e., non-stationary EIV, marked data and independent output) have been compared with a naive method which ignores the EIV issue and have been shown to yield superior performance for the intended noise cancellation problem.

\section{ACKNOWLEDGEMENTS}

We would like to acknowledge J. Lee, M. Downey, R. Turner and A. Maddever, from BHP-Billiton for their contribution to this project. We especially thank J. Lee for proposing the problem and M. Downey for collecting the experimental data used in this paper.

\section{REFERENCES}

Geophysical exploration for engineering and environmental investigations, 1998. Technical engineering and design guides as adapted from the U.S. Army Corps of Engineers No. 23. ASCE Press.

J. C. Agüero and G. C. Goodwin. Identifiability of EIV dynamic systems with non-stationary data. To be presented at the 17th IFAC World Congress, 2008a.

J. C. Agüero and G. C. Goodwin. Identifiability of errors in variables dynamic systems. Automatica, 44(2):371382, 2008b.

R. A. Carter. Exploration by the numbers. Engineering and Mining Journal, 206(5):34-38, 2005. URL wWw. scopus. com.

P. Kearney, M. Brooks, and I. Hill. An introduction to geophysical exploration. Blackwell Science, Malden MA, 3rd edition, 2002.

K. Lau, J. H. Braslavsky, and G. C. Goodwin. Errors-invariables problems in transient electromagnetic mineral exploration. In Proceedings of the 46th IEEE Conference on Decision and Control, New Orleans, LA, USA, 2007.

I. Markovsky, A. Kukush, and S. Van Huffel. On errorsin-variables estimation with unknown noise variance ratio. In Preprints of the 14th IFAC symposium in System Identification, pages 172-177, Newcastle, Australia, 2006.

M. N. Nabighian and J. C. Macnae. Time domain electromagnetic prospecting methods in electromagnetic methods. In M. N. Nabighian, editor, Electromagnetic Methods in Applied Geophysics Vol. 2, Application, Part $A$, pages 427-520. Society of Exploration Geophysics, 1991.

T. Söderström. Errors-in-variables methods in system identification. In Preprints of the 14th IFAC symposium in System Identification, pages 1-19, Newcastle, Australia, 2006.

A. Wald. The fitting of straight lines if both variables are subject to error. Ann. Math. Stat. Series B., 11(3): 284-300, 1940. 